\title{
Channel Estimation based on Kalman Filtering with BER Reduction in MIMO-OFDM Systems
}

\author{
Amit Kapoor \\ Student \\ Department of Electronics and Communication \\ Lovely Professional University \\ Phagwara, Punjab, India
}

\author{
Ishan Khurana \\ Assistant Professor \\ Department of Electronics and Communication \\ Lovely Professional University \\ Phagwara, Punjab, India
}

\begin{abstract}
MIMO-OFDM is one of the emerging communication schemes which provide efficient communication with multicarrier modulation. MIMO technology uses spatial diversity technique by using multiple antennas at the transmitter and the receiver side. In MIMO systems, the data streams arriving from different path with different time are combined at the receiver side. OFDM is a modulation scheme that allows digital data to be efficiently and reliably transmitted over a radio channel even in multipath environments. The main idea of OFDM system is to modulate the input data symbol onto a group of subcarriers with predefined coefficients such that the generated ICI within the group will cancel each other. The major disadvantage of this approach is higher bit error rates. The channel estimation also plays an important role in MIMO-OFDM systems. There are number of channel estimation methods which have already been proposed for MIMO-OFDM systems. In the past years many techniques had been proposed to reduce bit error rate in MIMO-OFDM systems. In this paper, we are proposing the new technique to reduce bit error rate in MIMO-OFDM technology. The proposed technique is filtering technique under this technique we use KALMAN filter for reducing bit error rate. Using kalman filter, channel estimation is also done properly as compared with the true value.
\end{abstract}

Keywords: OFDM, MIMO, KALMAN, BIT ERROR RATE, FILTERING

\section{INTRODUCTION}

MIMO (Multiple Input Multiple Output) is a wireless technology that uses multiple transmitters and receivers to transfer more than one data at the same time in the same frequency band. MIMO technology takes the advantage of the radio wave system called multipath. In multipath, the transmitted information reflected from walls, ceilings, and other objects and reached the receiving antenna multiple times via different angles and at slightly different timing. For multipath signals travelling through different paths has their independent effect imposed by the channels which are path gain or loss, path frequency offset, change in angle and path time delay. MIMO is a form of spatial diversity technique [1]. Previously multipath caused interference and slows down the wireless signals but the MIMO technology take the advantage of multipath behavior by using multiple antennas at the transmitter and the receiver side with a spatial diversity technique. By using spatial diversity technique, it will increase the performance and range. MIMO makes antennas work better by applying them to combine the data streams arriving from different paths with different time interval which will increase the receiver signal capturing power Due to multipath propagation, the ISI (Inter Symbol Interference) occurs in which one symbol interferes with the other subsequent symbol. ISI can be removed using different kinds of equalizers [2]. The received signals at the receiver side will contain noise due to the distortion, interference from any other transmission, fading or the atmospheric noise. At the receiver side, more than one antennas are used which receives a weighted or filtered sum of the different transmitted signals. The digital demodulator or the equalizer module in MIMO systems will reconstruct these signals from the weighted sum of signals transmitted by different antennas. This can be done using the estimate which is provided by the channel estimator and the received signal. After that the demodulated signal is passed to the decoder which analyses the structure of the received bit pattern in order to detect and correct the error. As a result of using the MIMO system, the performance benefits are due to the spatial multiplexing gain, diversity gain and the array gain [3].

Orthogonal frequency division multiplexing is one of the multi-carrier modulation techniques that transmit signals through multiple carriers. These carriers (sub carriers) have different frequencies and they are orthogonal to each other. Basically OFDM is a combination of modulation and multiplexing [4]. The basic principle of multicarrier modulation is to divide the data stream, $\mathrm{d}$, into $\mathrm{N}$ parallel data streams with a reduced data rate of $\mathrm{d} / \mathrm{N}$. Each low rate data steam is then modulated on a separate narrow band subcarrier and summed together for transmission, thereby providing the same data rate as an equivalent single carrier system. At the receiver a set of filter banks separate the wideband signal into the original narrowband subcarriers for demodulation. The advantage of this structure over single carrier systems is that the extended symbol time makes the signal less susceptible to effects of the channel such as multipath propagation which introduces Inter Symbol Interference (ISI). Each sub channel will therefore experience flat fading reducing the equalization complexity in the receiver dramatically [4]. Orthogonal Frequency Division Multiplexing is a special form of multicarrier modulation which is mainly well-matched for broadcast over a dispersive channel. The different carriers are orthogonal to each other, which means they are totally independent of one another. This is achieved by placing the carrier exactly at the nulls in the modulation spectra of each other. A simple communication system consists of a transmitter end which send the data and a receiver end at which the data is received. Usually there received data is not the same as the data sent. Because of the noise present in the 
medium the signal gets affected and distortion is observed in the signal. Various modulation techniques are under taken in order to ensure that the signal sent is safely available at the receiver end [4]. OFDM is an alternative wireless modulation technology to CDMA. OFDM is a modulation scheme that allows digital data to be efficiently and reliably transmitted over a radio channel even in multipath environments [5].

A well-known problem of OFDM system is its sensitivity to frequency offset between the transmitted and received signal which is caused by Doppler shift in the channel. This carrier frequency offset causes loss of orthogonality between subcarriers and the signals transmitted on each carrier which are not independent of each other. This leads to the inter-carrier interference (ICI) in OFDM. Researchers have proposed various methods to overcome the ICI in OFDM systems. The previously approaches that have been used to reduce ICI are categorized as frequency-domain equalization, time-domain windowing, and the ICI self-cancellation (SC) scheme [6-7].

The first method to overcome the ICI is a self-cancellation scheme, in which redundant data is transmitted onto adjacent sub-carriers such that the ICI between adjacent sub-carriers cancels out each other at the receiver end [8]. The second method is the windowing technique. The other techniques which are Maximum Likelihood (ML) estimation and the Extended Kalman Filter (EKF) method statistically estimate the frequency offset and correct the offset using the estimated value at the receiver.

\subsection{MIMO-OFDM SYSTEM MODEL}

The Block diagram of MIMO-OFDM system is shown in Figure. 1. At the transmitter side, a serial data bit stream is mapped to a symbol stream by a modulator. Then, this serial symbol stream is converted into parallel sub-streams. Next, pilot symbols for the channel estimation are inserted into these parallel sub-streams, in the frequency-domain, prior to the OFDM modulation. The OFDM modulation is then implemented by performing the inverse discrete Fourier transform (IDFT). Each transmits antenna sends independent OFDM symbols. The use of cyclic prefix is to reduce the effect of ISI. After that bits are converted from parallel to serial and given to the antenna.

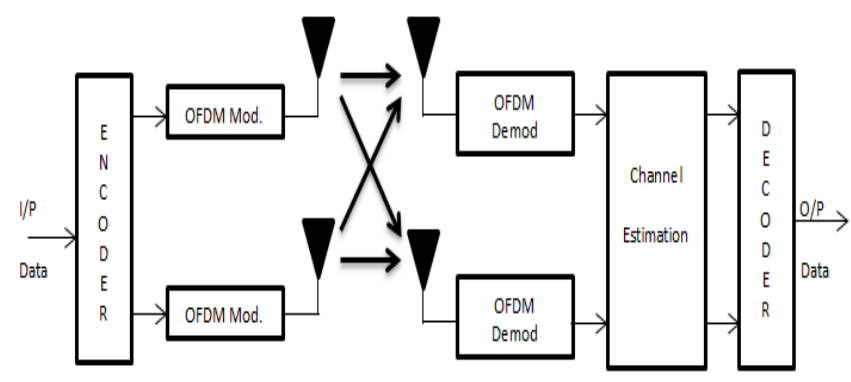

Figure.1 Block Diagram of MIMO-OFDM System model

At the receiver side the bits are converted from serial to parallel after getting from the antenna. Then cyclic prefix is removed which is added at the transmitter side and then OFDM mod again is performed in which DFT is performed. After this the channel estimation block comes in order to estimate the channel accurately. At last Demapping is done in order to get the desired output [9].

The received signal at receiver antenna can be expressed as:

$$
Y_{j}[n, k]=\sum H_{i j}[n, k] X_{i}[n, k]+N[n, k]
$$

(1)

Where $\mathrm{H}$ is the channel matrix, $\mathrm{X}$ is the input signal and $\mathrm{N}$ is noise. In the above equation, $i$ and $j$ denotes the transmitting antenna and receiving antenna, $\mathrm{n}$ and $\mathrm{k}$ are the time slot and the sub channel. The signal model can be expressed in matrix form as:

$$
\left[\begin{array}{c}
y_{1} \\
y_{2} \\
\vdots \\
y_{N}
\end{array}\right]=\left[\begin{array}{cccc}
h_{1,1} & h_{1,2} & \ldots & h_{1, N T} \\
h_{2,1} & h_{2,2} & \ldots & h_{2, N T} \\
\vdots & \vdots & \ddots & \vdots \\
h_{N R, 1} & h_{N R, 2} & \ldots & h_{N R, N T}
\end{array}\right]\left[\begin{array}{c}
x_{1} \\
x_{2} \\
\vdots \\
x_{N T}
\end{array}\right]+\left[\begin{array}{c}
N_{1} \\
N_{2} \\
\vdots \\
N
\end{array}\right]
$$

\subsection{Channel Estimation in MIMO-OFDM}

Channel estimation plays an important role in MIMO-OFDM systems. It is an important part of communication system. There are number of channel estimation methods which have already been proposed for MIMO-OFDM systems. A number of channel estimation methods have been proposed for MIMO-OFDM systems which can be broadly classified into three classes: the training based method, the blind method and semi blind method (combination of the first two methods). Channel estimation over rapidly time varying multipath fading channels has been considered in the papers. The Doppler spread information is also utilized for computing the frequency and time domain channel correlations in the channel estimation process [10].

The present channel estimation methods are divided into two types: based on the pilots and the second is the blind channel estimation which does not use pilots. Blind channel estimation methods do not use pilots and have higher spectral efficiency. Blind channel estimation methods are not suitable for applications with fast varying fading channels. The channel estimation methods which are widely used for the pilot aided channel estimation methods are divided into two types: the block type pilot channel estimation and the comb type pilot channel estimation [11]. In the block type pilot channel estimation, pilots are inserted into all the subcarriers of one OFDM symbol with a certain period and they can be adopted in slow fading channel which means the channel is static within a certain period of OFDM symbols. The comb-type refers to the pilots which are inserted at some specific subcarriers in each OFDM symbol. The comb-type is preferred in fast varying fading channel that is the channel varies over two adjacent OFDM symbols but remains static with one OFDM symbol [11]. When the fading channel cannot be viewed as a static within an OFDM symbol, then ICI occurs whereas the comb-type pilot patterns cannot eliminate ICI. There are some channel estimation methods for the pilot aided channel estimation which are LS (Least Square) and MMSE (Minimum Mean Square Estimation) [11].

\section{KALMAN FILTER}

In radio communication systems, filtering is a desirable factor. As radio communication signals are often corrupted with noise, a good filtering algorithm is required to remove noise from electromagnetic signals while retaining the useful information. Kalman Filtering is an effective method to filter impurities in linear systems. A Kalman filter is an optimal estimator infers parameters of interest from indirect, inaccurate and uncertain observations. The Kalman filter is a linear, discrete time, finite dimensional time-varying system 
that evaluates the state estimate that minimizes the meansquare error. The filter is very powerful in several aspects: it supports estimations of past, present, and even future states, and it can do so even when the precise nature of the modelled system is unknown. It might seem strange that the term filter would apply to an estimator. More commonly, a filter is a physical device for removing unwanted fractions of mixtures. Originally, a filter solved the problem of separating unwanted components of gas, liquid and solid mixtures. Kolmogorov and Wiener used the statistical characterization of their probability distributions in forming an optimal estimate of the signal, given the sum of the signal and noise. With Kalman filtering the term assumed a meaning that is well beyond the original idea of separation of the components of a mixture. It has also come to include the solution of an inversion problem, in which one knows how to represent the measurable variables as functions of the variables of principal interest. In essence, it inverts this functional relationship and estimates the independent variables as inverted functions of the dependent (measurable) variables. These variables of interest are also allowed to be dynamic, with dynamics that are only partially predictable [13].

The kalman filter basically consists of a set of mathematical equations that provides an efficient computational means to estimate the state of a process that minimizes the mean of the squared error. It operates recursively on streams of noisy input data to produce statistically optimal results the filter is very powerful in several aspects: it supports estimations of past, present, and even future states, and it can do so even when the precise nature of the modeled system is unknown [12]. In the Figure 2 the general filtering problem is explained in which the general filter simplifies to a kalman filter.

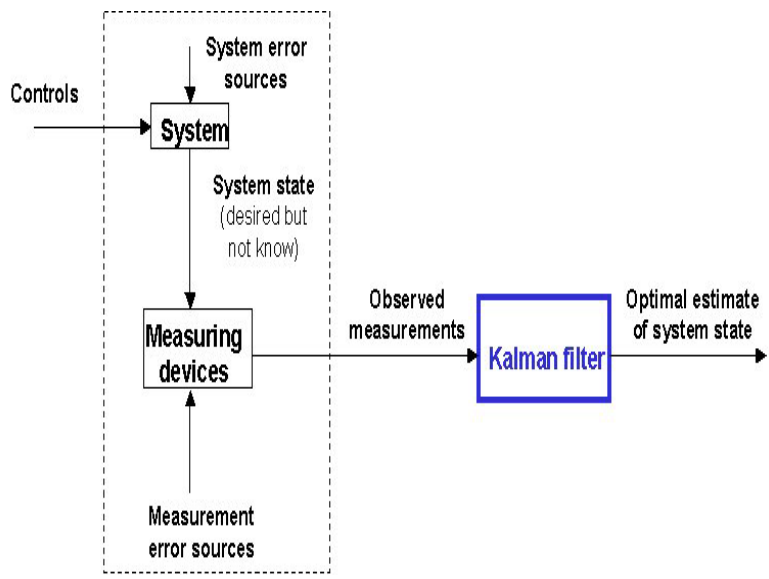

Figure.2 The general filtering problem

A physical system, (e.g., a mobile robot, a chemical process, a satellite) is driven by a set of external inputs or controls and its outputs are evaluated by measuring devices or sensors, such that the knowledge on the system's behavior is solely given by the inputs and the observed outputs. The observations convey the errors and uncertainties in the process, namely the sensor noise and the system errors. Based on the available information (control inputs and observations) it is required to obtain an estimate of the system's state that optimizes a given criteria. This is the role played by a filter. The system represented in Figure 2 has a linear-varying dynamics i.e.

$$
\begin{aligned}
& x_{k+1}=A_{k} x_{k}+B_{k} u_{k}+G w_{k} \\
& y_{k}=C_{k} x_{k}+v_{k}
\end{aligned}
$$

Where $\mathrm{x}(\mathrm{k}), \mathrm{u}(\mathrm{k}), \mathrm{w}(\mathrm{k}), \mathrm{v}(\mathrm{k}), \mathrm{y}(\mathrm{k})$ are real and $\mathrm{w}(\mathrm{k}), \mathrm{v}(\mathrm{k})$ are sequences of white, zero mean, Gaussian noise with zero mean

$$
E\left[w_{k}\right]=E\left[v_{k}\right]=0,
$$

And joint covariance matrix

$$
E\left[\left(\begin{array}{c}
w_{k} \\
v_{k}
\end{array}\right)\left(w_{k}^{T} v_{k}^{T}\right)\right]=\left[\begin{array}{cc}
Q_{k} & 0 \\
0 & R_{k}
\end{array}\right]
$$

The initial state, $x_{0}$ is a Gaussian random vector with mean

$$
E\left[x_{0}\right]=\bar{x}_{0}
$$

And covariance matrix

$E\left[\left(x_{0}-\bar{x}_{0}\right)\left(x_{0}-\bar{x}_{0}\right)^{T}\right]=\sum_{0}$

The sequence $\left\{u_{k}\right\}$ is deterministic.

The kalman filter is the filter that obtains the minimum mean square state error estimate. In fact, when $x(0)$ is a Gaussian vector, the state and observations noises $w(k)$ and $v(k)$ are white and Gaussian and the state and observation dynamics are linear.

The conditional probability density function $p\left(x_{k}\right) \mid\left(Y_{1}^{k}, U_{0}^{k-1}\right)$ are Gaussian for any $\mathrm{k}$. The mean, the mode and the median of this conditional pdf coincide. The kalman filter, i.e. the filter that propagates the conditional pdf $p\left(x_{k}\right) \mid\left(Y_{1}^{k}, U_{0}^{k-1}\right)$ and obtains the state estimate by optimizing a given criteria, is the best filter among all the possible filter types and it optimizes any criteria that might be considered.

The state estimate $\hat{x}(k \mid k)$ is the conditional mean of the pdf and the covariance matrix $P(k \mid k)$ quantifies the uncertainty of the estimate,

$$
\begin{aligned}
& \hat{x}(k \mid k)=E\left[x(k) \mid Y_{1}^{k}, U_{0}^{k-1}\right] \\
& P(k \mid k)=E\left[(x(k)-\hat{x}(k \mid k))(x(k)-\hat{x}(k \mid k))^{T} \mid Y_{1}^{k}, U_{0}^{k-1}\right]
\end{aligned}
$$

Therefore, rather than propagating the entire conditional pdf, the kalman filter only propagates the first and second moments. The kalman filter propagates the Gaussian pdf $p\left(x_{k}\right) \mid\left(Y_{1}^{k}, U_{0}^{k-1}\right)$ and therefore the filter dynamics defines the general transition from $p\left(x_{k}\right) \mid\left(Y_{1}^{k}, U_{0}^{k-1}\right)$ to $p\left(x_{k+1}\right) \mid\left(Y_{1}^{k+1}, U_{0}^{k}\right)[12]$.

\section{NEW PROPOSED TECHNIQUE}

The new proposed technique is based on the filtering. The bit error rate can be more in OFDMs MIMO technology. In MIMO the signals can split into multiple signals in source side and these signals are transferred to destination individually. In destination multiple antennas are responsible for receiving the individual signals. When the spited signals are received at the destination, they are recombined to form 
the original signals. This approach increase the data transfer rate, but the bit error rate is increased due to network inferences. In the past years many techniques had been proposed to reduce bit error rate in MIMO. The proposed technique is mathematically approach.

In the proposed model, the Kalman Filter along with the concept of repetitive slots and Correlated Channel Mapping. The proposed model of the system will be at the receiver side as shown in the figure. At the receiver side after the FFT is performed then kalman filter will be used. The initial steps of the proposed system are same as of traditional MIMO OFDM network. Here the proposed work implemented just after performing the FFT blocks. After that the channel mapping and estimation will be performed. If the channel is not mapped properly some filtration is performed so that the mapping will be done accurately. After that signal modulation is performed. As we get a mapped modulated signal, divide the signal in repetitive slots with some boundary definition.

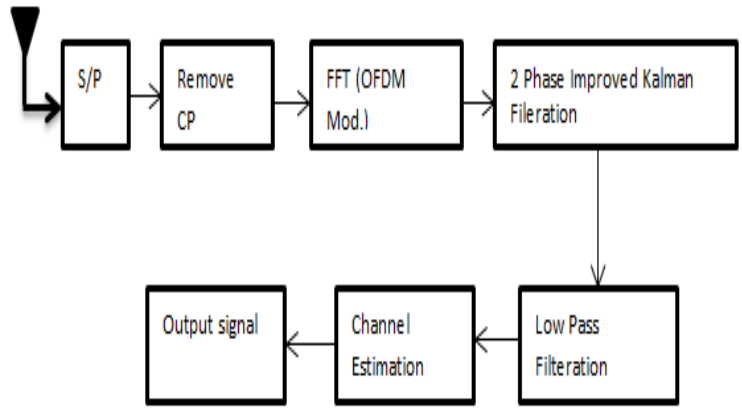

Figure.3 Block Diagram of proposed model

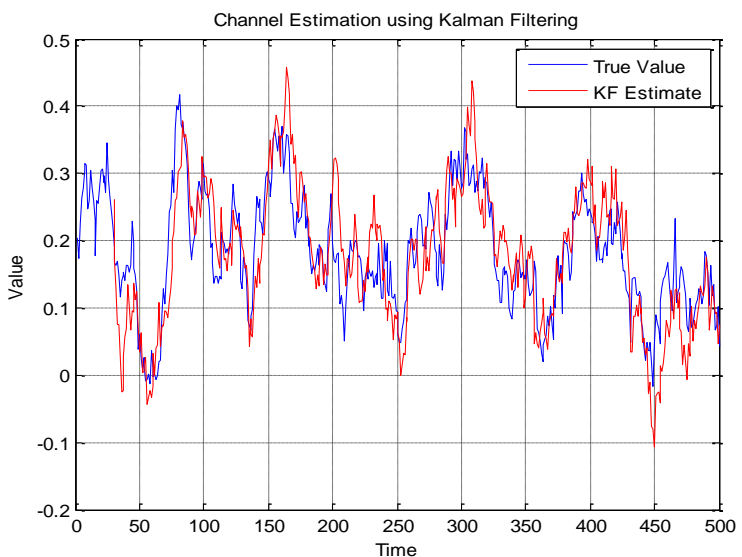

Figure.4 Channel estimation using Kalman Filter

In the figure 4 , the channel estimation is done using kalman filter and the kalman filter estimate is compared with the true value and it is observed that $\mathrm{KF}$ estimate is better. In the figure 5 and figure 6 below, the red line in the graph shows that the bit error rate is calculated in MIMO-OFDM system and blue line in the graph shows that the bit error rate is calculated in MIMO-OFDM system using filtering technique i.e. kalman filter.

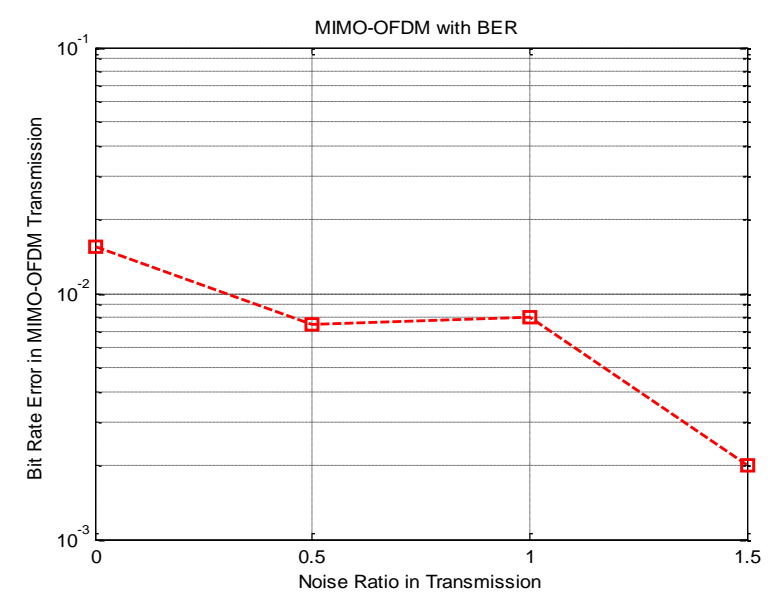

Figure.5 Signal to noise ratio in MIMO-OFDM transmission

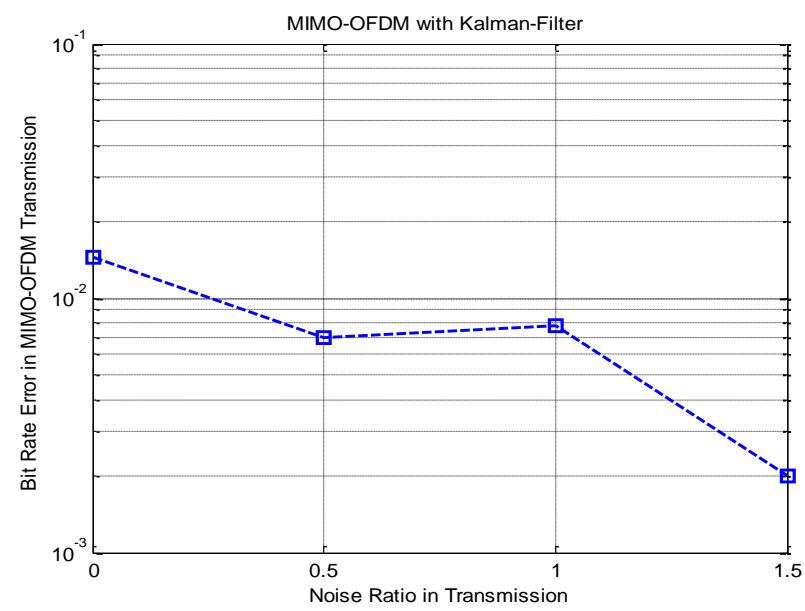

Figure.6 Signal to noise ratio in MIMO-OFDM transmission using KALMAN Filter

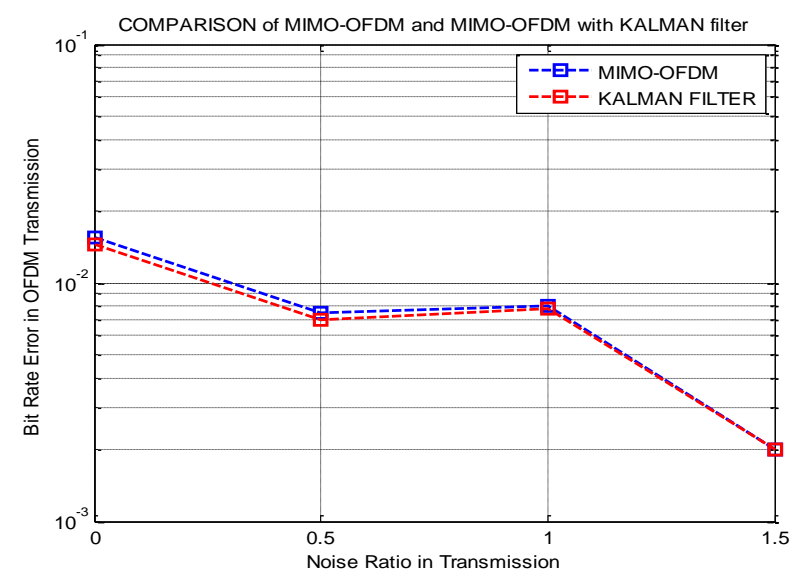

Figure.7 Comparison between SNR v/s BER in MIMOOFDM and with KALMAN filter

As illustrate in figure 7 , the blue line shows noise ratio in MIMO-OFDM and green line shows noise ratio in MIMOOFDM with KALMAN Filter. It shows that the SNR is improved using kalman filter in MIMO-OFDM systems. 


\section{CONCLUSION}

In this paper we conclude that the MIMO-OFDM is the efficient technology for data transfer at higher bit rate. Due to the nature of MIMO higher bit error rate had reduce the efficiency of the network. In this paper new filtering technique is purposed this technique is the mathematically technique in which we use KALMAN filter for reducing the bit error rate. This filtering technique is a mathematically technique in which Kalman filter is used for improving the performance.

The simulation results show that the two phase Kalman filter technique is more efficient as compared to the MIMO-OFDM systems and provide better bit error rate performance. Channel estimation is also improved with the help of Kalman filter and the simulation results shows that in Kalman Filter (KF) estimate, channel is estimated better. The simulations results shows that the bit error rate in MIMO-OFDM system using two phase Kalman filter has improved the SNR 0.046 $\mathrm{db}$. The future scope of this work is that bit error rate can be minimized by using other types of filter like adaptive filter and wiener filter. Using Kalman filter, bit error rate with different modulations can be examined.

\section{REFERENCES}

[1] Theodore S.Rappaport, "Wireless $\underset{\text { nd }}{\text { Communication: }}$ Principles and Practice", Prentice-Hall, 2 Edition, 2010, India.

[2] Yong Soo Cho, Jaekwon Kim Won Young Yang, ChungGu-Kang, "MIMO-OFDM Wireless Communication with MATLAB”, John Willey \& Sons (Asia) Pte Ltd.

[3] A.J. Paulraj, D. A. Gore, R.U. Nabar, H. Bolcskei, "An overview of MIMO communications - a key to gigabit wireless," Proceedings of the IEEE , vol.92,pp. 198-218, Feb. 2004.

[4] Alireza, Gary J. Saulnier, Member IEEE, "General ICI cancellation Scheme for OFDM Systems", IEEE transactions on vehicular technology, vol. 54, no. 1 , january 2005.

[5] Lajos L. Hanzo "OFDM and MC-CDMA" Willy Press.

[6] Anastasois Stamoulis, Suhas N. Diggavi, Member IEEE, " Intercarrier Interference in MIMO-OFDM", IEEE transactions on signal processing, vol. 50 , no. 10 , october 2002.

[7] J. Armstrong(1999), "Analysis of new and existing methods of reducing intercarrier interference due to carrier frequency offset in OFDM," IEEE Transactions on Communications, vol. 47, pp. 365 - 369, March 1999.

[8] Zhao and S. Haggman (2001), "Intercarrier interference self cancellation scheme for OFDM mobile communication systems," IEEE Transactions on Communications, vol. 49, pp. 1185 - 1191, July 2001.

[9] Mitalee Agrawal (2011)," BER Analysis of MIMO OFDM System for AWGN \& Rayleigh Fading Channel", International Journal of Computer Applications (0975 8887), Vol 34, pp 33-37, November 2011

[10] K.Vidhya R.Shankar kumar, " Channel Estimation Techniques for OFDM Systems", Proceedings of the International Conference on Pattern Recognition, Informatics and Medical Engineering, March 21-23, 2012.

[11] Neda Aboutorab, Wibowo Hardjawana and Branka Vucetic, Channel Estimation and ICI Cancellation for High Mobility Pilot-Aided MIMO-OFDM Systems, IEEE, 2013.

[12] M. Huang, X. Chen, L. Xiao, S. Zhou and J. Wang, "Kalman filter based channel estimation for OFDM in time-varying channels", IET Commun., 2007, 1, (4), pp.795-801.

[13] Hussein Hijazi (2010)," Joint Data QR-Detection and Kalman Estimation for OFDM Time-Varying Rayleigh Channel Complex Gains", IEEE Transactions On Communications IEEE, 2010 